\title{
Development of a tiered and binned genetic counseling model for informed consent in the era of multiplex testing for cancer susceptibility
}

\author{
Angela R. Bradbury, MD ${ }^{1-3}$, Linda Patrick-Miller, PhD ${ }^{4,5}$, Jessica Long, MS, CGC1, \\ Jacquelyn Powers, MS, CGC ${ }^{1}$, Jill Stopfer, MS, CGC ${ }^{1}$, Andrea Forman, MS, CGC ${ }^{6}$, \\ Christina Rybak, MS, $\mathrm{CGC}^{6}$, Kristin Mattie, MS, CGC ${ }^{7}$, Amanda Brandt, MS, CGC ${ }^{1}$, \\ Rachelle Chambers, MS, CGC ${ }^{4}$, Wendy K. Chung, MD, PhD ${ }^{8,9}$, Jane Churpek, MD ${ }^{4}$, \\ Mary B. Daly, MD, PhD ${ }^{6}$, Laura Digiovanni, MLA ${ }^{1}$, Dana Farengo-Clark, MS, $\mathrm{CGC}^{7}$, \\ Dominique Fetzer, BA ${ }^{1}$, Pamela Ganschow, MD ${ }^{10}$, Generosa Grana, MD, FACP7, \\ Cassandra Gulden, MS, $\mathrm{CGC}^{4}$, Michael Hall, MD', Lynne Kohler, BA ${ }^{1}$, \\ Kara Maxwell, MD, PhD' ${ }^{1}$, Shana Merrill, MS, CGC ${ }^{11}$, Susan Montgomery, BSN, OCN ${ }^{6}$, \\ Rebecca Mueller, MS, CGC ${ }^{1}$, Sarah Nielsen, MS, CGC4, Olufunmilayo Olopade, MD, FACP ${ }^{4,5}$, \\ Kimberly Rainey, MS, $\mathrm{CGC}^{6}$, Christina Seelaus, MS, $\mathrm{CGC}^{10}$, Katherine L. Nathanson, MD ${ }^{3,11}$ \\ and Susan M. Domchek, MD ${ }^{1,3}$
}

\begin{abstract}
Purpose: Multiplex genetic testing, including both moderate- and high-penetrance genes for cancer susceptibility, is associated with greater uncertainty than traditional testing, presenting challenges to informed consent and genetic counseling. We sought to develop a new model for informed consent and genetic counseling for four ongoing studies.
\end{abstract}

Methods: Drawing from professional guidelines, literature, conceptual frameworks, and clinical experience, a multidisciplinary group developed a tiered-binned genetic counseling approach proposed to facilitate informed consent and improve outcomes of cancer susceptibility multiplex testing.

Results: In this model, tier 1 "indispensable" information is presented to all patients. More specific tier 2 information is provided to support variable informational needs among diverse patient populations. Clinically relevant information is "binned" into groups to minimize information overload, support informed decision making, and facilitate adaptive responses to testing. Seven essential elements of informed consent are provided to address the unique limitations, risks, and uncertainties of multiplex testing.

Conclusion: A tiered-binned model for informed consent and genetic counseling has the potential to address the challenges of multiplex testing for cancer susceptibility and to support informed decision making and adaptive responses to testing. Future prospective studies including patient-reported outcomes are needed to inform how to best incorporate multiplex testing for cancer susceptibility into clinical practice.

Genet Med advance online publication 9 October 2014

Key Words: genetic counseling; genetic testing for cancer susceptibility; informed consent; multiplex genetic testing

\section{INTRODUCTION}

Genetic screening for cancer susceptibility is one application of "personalized medicine" and has become an evidence-based medical practice that improves patient survival. ${ }^{1}$ However, many individuals and families suspected to be at a high risk for cancer, based on family or personal medical histories, are not found to have mutations in the most common susceptibility genes. For example, many women in whom genetic susceptibility for hereditary breast and ovarian cancer is suspected do not have a BRCA1/2 mutation. Testing of multiple high-penetrance genes simultaneously has long been an accepted practice for susceptibility to colorectal cancer (e.g., mismatch repair genes in Lynch syndrome) and pheochromocytoma (e.g., succinate dehydrogenase complex genes). Since 2012, multiplex (i.e., multi-gene) panels, which include both high- and moderate-penetrance cancer susceptibility genes, have emerged for many other

\footnotetext{
${ }^{1}$ Division of Hematology-Oncology, Department of Medicine, University of Pennsylvania, Philadelphia, Pennsylvania, USA; ${ }^{2}$ Department of Medical Ethics and Health Policy, University of Pennsylvania, Philadelphia, Pennsylvania, USA; ${ }^{3}$ Abramson Cancer Center, University of Pennsylvania, Philadelphia, Pennsylvania, USA; ${ }^{4}$ Division of HematologyOncology, Department of Medicine, The University of Chicago, Chicago, Illinois, USA; ${ }^{5}$ Center for Clinical Cancer Genetics and Global Health, The University of Chicago, Chicago, Illinois, USA; ${ }^{6}$ Department of Clinical Genetics, Fox Chase Cancer Center, Philadelphia, Pennsylvania, USA; ${ }^{7}$ Department of Hematology/Oncology, MD Anderson Cancer Center at Cooper, Camden, New Jersey, USA; ${ }^{8}$ Department of Pediatrics, Columbia University Medical Center, New York, New York, USA; ${ }^{9}$ Department of Medicine, Columbia University Medical Center, New York, New York, USA; ${ }^{10}$ Department of Internal Medicine, John H. Stroger, Jr. Hospital, Chicago, Illinois, USA; ${ }^{11}$ Department of Translational Medicine and Human Genetics, University of Pennsylvania, Philadelphia, Pennsylvania, USA. Correspondence: Angela R. Bradbury (Angela.bradbury@uphs.upenn.edu)
} 
cancer types, including breast and ovarian, and they are currently being used in clinical practice despite questions regarding their clinical utility. ${ }^{2-14}$

Multiplex testing for cancer susceptibility represents the broader transition in clinical genetics from sequential limited (e.g., single-gene) evaluation to multiplex, parallel sequencing of many genes and, in some settings, whole-exome sequencing. This transition from limited (e.g., single gene) to bundled (i.e., multiplex panels) or broad (e.g., whole-exome sequencing) genomic applications presents many challenges. ${ }^{15,16}$ Many of the genes included on "cancer gene panels" are moderatepenetrance genes, increasing cancer risk by two- to fourfold, and their clinical utility remains unclear. ${ }^{10,13,17,18}$ The risks, benefits, and clinical utility of testing for multiple genes of varied risk, cancer spectrum, and clinical utility are unknown and present challenges to traditional approaches to informed consent for genetic testing. ${ }^{8,15,18-20}$ Multiplex testing has the potential to increase anxiety, uncertainty, and potentially the adoption of inappropriate screening or risk-reducing surgeries. Effective genetic education and counseling are designed to minimize these risks and enhance adaptive responses to genetic risk. ${ }^{21}$ Traditional counseling models, although intended to be person-centered or individualized, ${ }^{21-23}$ are not designed to provide comprehensive information for simultaneous testing of multiple genes with diverse cancer spectra, risks, and medical management strategies. Thus, there is an urgent need to develop and evaluate new approaches to genetic counseling and informed consent with the clinical implementation of multiplex testing for cancer susceptibility, ${ }^{2,6-8,15,18-20}$ and to study the risks and benefits of bundled tests over sequential, discrete testing. ${ }^{16}$

Here we describe the development of a new model for the delivery of multiplex testing for cancer susceptibility. This model was developed for four related but distinct studies evaluating the outcomes of multiplex genetic testing for breast cancer susceptibility (Table 1), in which the traditional model for cancer genetic counseling would not be feasible and could be overwhelming and could negatively impact testing decisions and outcomes. Thus, a new and standardized approach to the informed consent and counseling process across genetic counselors and sites is needed to assess counseling fidelity and evaluate participant outcomes over time. Additionally, this model was developed to be broadly applicable to multiplex testing in other clinical settings in which panels contain genes associated with varying disease risk, health implications, or clinical utility.

\section{MATERIALS AND METHODS}

A multidisciplinary working group of study investigators and clinical genetic providers from six collaborating sites

Table 1 Characteristics of studies incorporating multiplex testing for breast cancer susceptibility

\begin{tabular}{|c|c|c|c|c|}
\hline & $\begin{array}{l}\text { COGENT study } \\
\text { (Communication of } \\
\text { Genetic Test Results } \\
\text { by Telephone) }\end{array}$ & $\begin{array}{l}\text { RESPECT study (Returning Genetic } \\
\text { Research Panel Results } \\
\text { for Breast Cancer Susceptibility) }\end{array}$ & $\begin{array}{l}\text { METEOR pilot study } \\
\text { (Multiplex Testing } \\
\text { for Evaluation of Breast } \\
\text { Cancer Risk) }\end{array}$ & $\begin{array}{c}\text { METEOR longitudinal study } \\
\text { (Multiplex Testing } \\
\text { for Evaluation of Breast } \\
\text { Cancer Risk) }\end{array}$ \\
\hline $\begin{array}{l}\text { Study } \\
\text { design, } \\
\text { measures, } \\
\text { participants, } \\
\text { and } \\
\text { procedures }\end{array}$ & $\begin{array}{l}\text { Randomized } \\
\text { longitudinal study of } \\
\text { in-person (standard- } \\
\text { of-care) and telephone } \\
\text { disclosure of clinical } \\
\text { genetic test results. } \\
\text { Adapted for inclusion } \\
\text { of multiplex testing } \\
\text { April 2014. Clinic-based } \\
\text { patients complete } \\
\text { baseline, postdisclosure, } \\
\text { and 6- and } 12 \text {-month } \\
\text { surveys }\end{array}$ & $\begin{array}{l}\text { Observational longitudinal study of } \\
\text { BRCA1/2-negative research participants } \\
\text { presented with the opportunity to } \\
\text { receive research multiplex results. } \\
\text { Participants complete in-person or } \\
\text { telephone pretest counseling to } \\
\text { understand the risks/benefits and } \\
\text { limitations of learning individual } \\
\text { research results. Participants complete } \\
\text { baseline (before pretest counseling), } \\
\text { after pretest counseling, postdisclosure, } \\
\text { and 6- and } 12 \text {-month surveys }{ }^{b}\end{array}$ & $\begin{array}{l}\text { Observational pilot study of } \\
\text { patients who meet eligibility for } \\
\text { METEOR longitudinal (BRCA1/2 } \\
\text { negative) or COGENT (BRCA1/2 } \\
\text { naive) studies. Patients } \\
\text { complete in-person pre- and } \\
\text { posttest counseling. Patients } \\
\text { and genetic providers complete } \\
\text { mixed-method surveys at } \\
\text { baseline (before pretest } \\
\text { counseling), after pretest } \\
\text { counseling, and postdisclosure }\end{array}$ & $\begin{array}{l}\text { Observational longitudinal study } \\
\text { of } B R C A 1 / 2 \text { negative clinical } \\
\text { patients presented with the } \\
\text { opportunity to receive clinical } \\
\text { multiplex testing for other } \\
\text { high- and moderate-penetrance } \\
\text { genes. Patients complete } \\
\text { in-person pretest counseling } \\
\text { and baseline (before pretest } \\
\text { counseling), after pretest } \\
\text { counseling, postdisclosure, and } \\
6 \text { - and } 12 \text {-month surveys }{ }^{\mathrm{b}}\end{array}$ \\
\hline Funding & $\begin{array}{l}\text { National Cancer Institute } \\
\text { (R01 CA160847) }\end{array}$ & $\begin{array}{l}\text { American Society of Clinical Oncology } \\
\text { Conquer Cancer Foundation and the } \\
\text { Breast Cancer Research Foundation }\end{array}$ & $\begin{array}{l}\text { Rooney Family Fund; Myriad } \\
\text { Genetics provides the cost of } \\
\text { multiplex testing }\end{array}$ & $\begin{array}{l}\text { Rooney Family Fund; Myriad } \\
\text { Genetics provides the cost of } \\
\text { multiplex testing }\end{array}$ \\
\hline
\end{tabular}

${ }^{\mathrm{a} C}$ ClinicalTrials.gov NCT01736345. ${ }^{\mathrm{b}} \mathrm{Common}$ outcomes and measures include understanding of genetic information (knowledge and perceptions of genetic disease ${ }^{\mathrm{a}}$ ), reactions to genetic information (psychological distress including generala and event-relateda anxiety and depressiona, cancer-specific worrya, and satisfaction with genetic

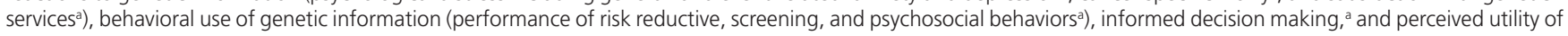
genomic information (novel measure). 
participated in three conference calls (August-September 2013) to develop a new model for informed consent and genetic counseling for four ongoing studies evaluating the outcomes of multiplex genetic testing for breast cancer susceptibility. This multidisciplinary group of investigators collaborating on related research (see Table 1) includes physicians and genetic counselors with expertise in cancer genetics and medical genetics, as well as individuals with expertise in clinical psychology, health communication, and medical ethics. Multiple drafts of the proposed model, counseling checklists, and the manuscript were circulated for comments, reviewed, and iteratively revised (October 2013-May 2014).

\section{Rationale for a tiered-binned approach to pretest counseling for multiplex testing}

In cancer genetics, promoting informed consent for genetic testing is traditionally facilitated through pretest counseling with a genetic or other adequately trained professional. This is achieved through "comprehensive" education and pretest counseling, including genetic education, specific disease risks and medical management options for individual genes, and the potential medical and psychosocial implications of testing, highlighting the option to decline testing and consider alternative options for risk assessment and risk management. ${ }^{19,21,23-27}$ Effective pretest education and counseling can prepare patients for uncertain results, increase understanding, and decrease test-related anxiety and negative responses to uncertainty. ${ }^{21}$ It is increasingly recognized that the traditional standard of "comprehensive" or "specific" informed consent for cancer susceptibility testing will not be feasible, applicable, or beneficial to patients as we move to broader genetic testing, which includes genes of varying risks and clinical implications (e.g., the finding of incidental mutations in noncancer genes of high penetrance). ${ }^{15,28,29}$ "Generic" consent has been proposed as an alternative strategy to provide patients with minimally sufficient information to make informed decisions regarding testing or receipt of genetic information..$^{26,30}$ The "layered" approach to generic consent includes identifying broad concepts and common-denominator elements required for all patients. We propose that this approach, originally proposed for prenatal screening and whole-exome sequencing, can support informed consent in the setting of multiplex testing for cancer susceptibility and enhance adaptive responses to testing, particularly in the era of bundled and broad testing and variable clinical utility. ${ }^{29}$ A layered or tiered approach to education and counseling is utilized in which tier 1 , "indispensable" information is presented to all patients and tier 2 information provides additional detail as needed, supporting variable information needs among diverse patient populations..$^{29,30}$

An additional strategy to address the large amount of potentially relevant information generated with broad genetic testing is to organize the information into clinically relevant "bins." 28 This approach was proposed as a framework to organize genes to consider the return of incidental findings. ${ }^{28}$ As suggested by others, binning genes could also be a useful approach for consent and patient education. ${ }^{20,28}$ Rather than providing specific risks for each individual gene, genes can be organized into bins, defined by their risk, clinical utility, evidence supporting medical management, or other relevant features, for example, "some genes on this panel have very high lifetime risks for cancers that lack effective surveillance options, so prophylactic surgery such as removal of the ovaries or the stomach may be recommended as a possible method to reduce the risk for cancer," or "some genes have very limited information available regarding risks for cancer, but more may be known in the future." Some patients may desire additional specific information, which can be shared in an individualized fashion as tier 2 information. We propose that binning and tiered strategies can be combined effectively as a new approach to informed consent and genetic counseling in the era of multiplex genetic testing and present additional key components to the successful implementation of this approach for the research setting and, ultimately, clinical care.

\section{RESULTS}

\section{A tiered-binned approach to multiplex testing for cancer susceptibility}

We used the American Society of Clinical Oncology 14 Basic Elements of Informed Consent, consistent with other practice standards, as the starting framework. ${ }^{26,27}$ Through deliberation, we identified several of the traditional basic elements that could be overwhelming to patients if presented in comprehensive detail and were amenable to presentation in a tiered and binned fashion (Table 2). These elements included: (i) information on specific genes being tested and the associated risk; (ii) specific implications of positive, negative, and uninformative results; (iii) options and limitations of medical management or riskreducing strategies; and (iv) implications for family members. For these elements, we identified key common-denominator themes and the important minimum elements that patients must understand in order to make an informed decision in the setting of multiplex testing (Table 2). In addition, patients are informed that more specific and comprehensive information can be provided in result disclosure sessions. Several basic elements of informed consent are relevant to cancer susceptibility testing regardless of the type of testing (e.g., limited or multiplex testing). These elements are not significantly modified from traditional counseling practice, although some key differences with multiplex testing are reflected in the key elements outlined here. As an example, counseling checklists for pretest and posttest counseling in the METEOR studies (see Table 1) are included as Supplementary Appendixes online. Checklists for the RESPECT study include modifications to address studyspecific contexts (e.g., the difference between research and clinical testing). Additional previously published modifications developed for telephone communication ${ }^{31}$ (e.g., standard and situational probes to address lack of visual cues with telephone and challenges with distractions and disruptions) are also included for COGENT and RESPECT checklists.

One area of debate within the working group was the need to provide patients with specific examples of scenarios in which 
Table 2 Adaptations and key themes in the tiered-binned model for multiplex testing

\section{Fourteen traditional key elements of informed consent for cancer susceptibility testing (ASCO guidelines 2010 ${ }^{\mathrm{a}}$ )}

1. Basic genetic education

2. Information on the specific genetic mutation (s) or genomic variant (s) being tested, including whether the range of risk associated with the variant will impact medical care, and associated risks (\#1)

3. Implications of a positive, negative, and VUS results (\#2)

4. Possibility that the test will not be informative (\#3)

5. Options and limitations of medical surveillance and strategies for prevention after genetic or genomic testing (\#12)

6. Risk of passing a genetic variant to children (\#5)

7. Importance of sharing genetic and genomic test results with at-risk relatives so that they may benefit from this information (\#13)

8. Psychological implications of test results (benefits and risks) (\#8)

9. Options for risk estimation without genetic or genomic testing (\#4)

10. Technical accuracy of the test and associated fees (\#6, 7)

11. Privacy, risks, and protections against genetic discrimination and possible use of DNA testing samples in future research $(\# 9,10,11)$

12. Plans for follow-up after testing (\#14)

\section{Adaptations in the} tiered-binned model

Consistent with traditional practice

Binned and tiered information on the genes being tested and the impact on medical care ${ }^{a}$

Binned and tiered information on types of test results and the impact on medical recommendations ${ }^{\mathrm{a}}$ options and limitations of medical surveillance and strategies for prevention after genetic or genomic testing (e.g., prophylactic surgery versus early detection) ${ }^{2}$

Binned and tiered information regarding risks for children and family members $^{\mathrm{a}}$
Binned and tiered information on the

Consistent with traditional practice

Consistent with traditional practice

Key themes (tier 1 elements) in the tiered-binned model

1. Testing can identify varying risks (high, moderate, and uncertain) for a wide spectrum of cancers.

2a. Implications of results vary depending on the gene and result.

2b. Finding a mutation may or may not change your current medical care.

3. The evidence and support for medical recommendations varies by gene and result (e.g., in some cases there are evidence-based recommendations, others consensus guidelines, others no clear consensus or guidelines).

4a. Some genes are associated with risks for cancer in childhood, while others only confer risks in adulthood. Some genes are associated with disorders other than cancer.

$4 b$. For some genes, there is currently insufficient information to recommend testing for relatives.

5. There is the potential for various uncertainties (e.g., uncertain results, uncertain risks, and uncertainty regarding medical management options).

6. Given a range of testing options and variable risks, benefits, and utilities, patients should be aware that testing is a choice.

Consistent with traditional practice

Consistent with traditional practice

Consistent with traditional practice
7. Risk estimates and cancer spectrum may be revised over time. Ongoing communication with the cancer genetics team will be beneficial, particularly in the setting of uncertain results or recommendations.

ASCO, American Society of Clinical Oncology; VUS, variants of uncertain significance.

allustrative examples can be included to represent specific examples (e.g., prophylactic removal of the stomach is considered for individuals who are at increased risk for stomach cancer and TP53 mutations are associated with high risks for a variety of cancers, some of which are hard to prevent or screen for, and that can occur in childhood).

patients have been known to have variable preferences for testing. ${ }^{32}$ For example, some members endorse a specific discussion of medical management strategies and potential psychosocial risks with identification of a germline TP53 mutation because the mutation confers risks for a wide range of cancers, some of which have no established or limited strategies for screening or risk reduction, or $\mathrm{CDH} 1$ mutations, for which prophylactic gastrectomy may need to be considered given limitations of screening for diffuse gastric cancer. Other members felt that specific examples are not necessary at pretest counseling and that the possibility of these uncertainties can be sufficiently shared in a tiered-binned model before testing, or in greater detail when relevant at posttest counseling. The group was not able to reach a consensus on this topic, highlighting an important area for continued deliberation and consideration in ongoing research.

Several additional strategies endorsed by patients and providers, informed by our team's multidisciplinary expertise and existing literature, are included in this model to improve patient understanding and satisfaction. ${ }^{22,23,33-35}$ Visual aids, strongly valued by patients, particularly in the context of complex medical information, ${ }^{16,36,37}$ are included to enhance understanding of complex information. Study-specific visual aids have been developed for each of the studies to address the goals, populations, and context of the specific study. Consistent with current practice, these are designed to be provided to participants in print format in the context of the counseling session. For 
patients receiving counseling by phone, visual aids are mailed to participants in advance of their counseling session so they can be viewed during the counseling session. A key novel component of this new approach is standardized provider communication probes, utilized in counseling sessions to assess desired levels of information (e.g., "As we go along, please tell me if you would prefer that we discuss this information in greater detail or if you have heard enough detail"), knowledge and genomic health literacy (e.g., "We have talked about a lot of things, what questions do you have for me now?"), and affect (e.g., "How are you feeling now that you know...?"). Of note, this strategy is utilized to address diverse patient populations in which genomic health literacy and informational needs vary. ${ }^{38}$ At the conclusion of the session, we include a "Teach Back" probe (e.g., "Please tell me in your own words your understanding of what we have discussed today and what that might mean for you") to assess comprehension and facilitate informed decision making, providing the opportunity for remediation of any misperceptions. ${ }^{39,40}$ This additional strategy is designed to help providers address varying genomic health literacy and support informed decision making as genetic testing is more widely disseminated and incorporated in diverse medical practices. ${ }^{38}$

\section{Tier 1 essential elements for informed consent with multiplex testing}

We identified seven essential minimal elements necessary for patients to make an informed decision regarding genetic testing options to include multiplex testing for cancer susceptibility and to enhance adaptive psychosocial and behavioral responses to multiplex testing (Table 2). We note that multiplex testing panels vary. Some may include only genes in which mutations confer a similarly high risk of the same cancers (e.g., mismatch repair genes and Lynch syndrome), and not all of these elements may need to be invoked depending on the particular panel.

1. Testing can identify varying risks (high, moderate, and uncertain) for a wide spectrum of cancers. Although some patients may desire detailed information for all or select genes, for many patients a comprehensive review might generate distress and negatively impact informed decision making. Nonetheless, it is important for patients to understand the general scope, variability, and range of risks and associated cancer types. We propose this be shared in a tiered-binned fashion based on individual informational preferences.

2. The implications of a positive or negative result will vary based on the particular gene, the personal and family history, and the prevalence of mutations in the general population (carrier rate). Given the current unknowns for many of the genes on multiplex panels, and the potential for unanticipated positive results in high-penetrance genes (where the personal and/or family history is not suggestive of the phenotype), the implications of results may be highly dependent on the family history. Further, patients should be prepared for uncertainty and the potential for no change in their medical management. Again, we propose this information can be shared in a tiered-binned fashion, and there may be a role for specific examples (e.g., TP53, CDH1) that could influence decision making or illustrate particular themes.

3. The evidence supporting medical recommendations for specific genes and results varies. In some cases, there is strong evidence to guide medical recommendations (e.g., $B R C A 1 / 2$, Lynch syndrome). For other genes, there are consensus guidelines (TP53). In many genes, there is very limited evidence to guide medical recommendations (e.g., CHEK2, ATM). Even in the setting of a deleterious mutation, patients' results might not change their medical management. Although we have traditionally discussed specific risk-reduction options (e.g., prophylactic oophorectomy, the addition of screening breast magnetic resonance imaging) in pretest counseling, we propose in the tiered-binned model that general strategies be presented to all patients (e.g., "Cancer risk may be addressed by increased screening, chemoprevention, or, in some cases, prophylactic surgeries"), with additional information provided based on personal and family history and informational assessments and requests.

4. Mutations in some genes are associated with a risk for cancer during childhood and adolescence and can be associated with conditions other than cancer. Additionally, cascade testing in other relatives may or may not be indicated, particularly for moderate-penetrance genes. The implications for family members are much more varied and complex as we transition to multiplex panels with overlapping phenotypes. The relevance of moderatepenetrance genes for relatives remains uncertain, and results may be context-specific and modified by the family history. We propose that in the tiered-binned model, the range of possibilities be presented to patients so they have a general understanding of the potential outcomes of testing, and, if interested, patients can engage in a more in-depth discussion.

5. There is the potential for various uncertainties (e.g., uncertain results, uncertain risks, and uncertainty regarding medical management options). Variants of uncertain significance (VUS) are possible and more frequent with panel testing than with single-gene or targeted testing. Although genetic providers are accustomed to discussing this possibility with patients, the frequency of VUS is significantly higher with panel testing (up to $30 \%)^{10,11,13,20}$ and could be associated with distress, misunderstanding, and inappropriate screening or risk-reducing surgeries. Patients need to be aware that VUS are a possibility, that VUS are not to be used to make medical management decisions, and that interpretation of results may be clarified over time. Understanding the possibility of VUS allows patients to make more informed decisions about proceeding with testing based on their tolerance 
for ambiguity, and is designed to minimize negative responses to testing for those who proceed.

6. Given a range of testing options and variable risks, benefits, and utilities, patients should be aware that testing is a choice. At this time there are various cancer susceptibility testing options for genetic providers and patients. These include standard limited (e.g., single-gene testing) and various multiplex panels ranging from those with high-penetrance genes only to panels focused on particular tumor types (e.g., BreastNext, ColoNext) and broader panels including high- and moderate-penetrance genes (e.g., MyRisk). ${ }^{8,20}$ At least one commercial laboratory is considering offering the option to select specific genes when ordering genetic testing, allowing patients and providers to more efficiently direct testing and limit the potential for uncertain or unwanted information. For most patients, limited testing for known syndromes (e.g., $B R C A 1 / 2$ and mismatch repair genes for families with breast, ovarian, or colon cancer) for which there is clear evidence guiding medical management recommendations remains a valid option. Choosing how large a gene panel to use depends, in part, on the urgency of testing (e.g., newly diagnosed patients undergoing testing to make a surgical decision) and the patient's tolerance of ambiguity regarding VUS and genes with as-yetundefined penetrance and cancer spectra. Also, testing for additional genes will remain an option in the future for patients who elect to defer multiplex testing initially.

7. Ongoing communication with the cancer genetics team will be critical, particularly in the setting of uncertain results or recommendations. At this time, the evidence base and best practice guidelines for risk estimation, interpretation, and medical management recommendations are still in development for many of the genes on these panels. ${ }^{6}$ Thus, multiplex testing for panels with high- and moderate-penetrance genes will be a collaborative learning process between the genetics team and patients because risk estimates, cancer spectra, and best practices for medical management will be revised over time. Best practices for longitudinal communication and provision of medical updates constitute a significant knowledge gap for the field given the higher rates of VUS and various current uncertainties. At minimum, follow-up requires patients or programs to maintain upto-date contact information. Some patients may benefit from regularly planned follow-up with a genetics team, which might be associated with access burdens for some patients.

\section{DISCUSSION}

As highlighted by many, the clinical availability of multiplex testing for common cancers, containing high- and moderatepenetrance genes, presents considerable challenges to the clinical implementation of inherited genetics. Among these challenges is protecting patient autonomy and limiting the psychosocial and medical risks of cancer susceptibility testing. To our knowledge, this model is the first proposed for informed consent and genetic counseling for multiplex testing including both high- and moderate-penetrance genes for cancer susceptibility. We present this model as a starting point for the purposes of ongoing studies and relevant fields to consider the merits and limitations of various approaches to informed consent and delivery of multiplex cancer genetic testing. Two key questions in the field remain regarding the relative advantages of bundling (i.e., multiplex testing) and the best model for delivering bundled and broad sequencing. We acknowledge that there will likely be alternative models that could be considered and compared to understand their relative benefits and risks.

This approach has several strengths, reflecting the consensus of a multidisciplinary team from six cancer genetics programs. These teams have utilized multiplex testing in the clinical and research settings and used strategies that have been proposed in other settings of broad genomic testing (e.g., prenatal and whole-exome sequencing) for which comprehensive approaches to counseling and consent are not feasible. Our tiered-binned approach is grounded in existing guidelines for cancer susceptibility testing and is intended to preserve key components of informed consent and pretest counseling while utilizing strategies to tailor the depth of information to individual needs and to organize content into key themes and elements to enhance understanding and informed decision making. Thus, we have included several adaptations and elements to facilitate patientcentered communication (e.g., assessments of cognitive and affective responses and informational needs)..$^{22,23,33-35}$ Equally important, we feel this model can provide a framework for other multiplex and broad genetic testing for disease susceptibility in other contexts. This model also has the potential to enhance the efficiency of genetic services given the increasing demand and a limited workforce.

Despite these strengths, we acknowledge several limitations. There is currently no clear standard delivery model for multiplex testing for cancer susceptibility, raising the question of what is the most appropriate comparison for innovations in the field. As new alternative counseling and delivery models are developed, studies designed to compare various delivery models could be valuable and ultimately define standards for the field. Our novel tiered-binned model was developed to inform several studies evaluating the outcomes of receipt of multiplex testing for cancer susceptibility; however, the outcomes of the tiered-binned approach are currently unknown. Data evaluating the informed choice and the cognitive, affective, and behavioral responses to this model of genetic counseling for multiplex testing for breast cancer susceptibility are the goal of the described ongoing studies. Although we believe this approach is consistent with the way in which many genetic providers are approaching clinical counseling for multiplex testing in the absence of any consensus guidelines, ${ }^{20}$ there are likely other valid approaches. As described above, our group debated the merits of providing specific information for particular genes (e.g., TP53, CDH1) when patients 
have expressed varying testing preferences. ${ }^{32}$ The value of utilizing specific examples remains unknown. There is also value in evaluating various visual formats and content of risk information, particularly among populations of varying genomic literacy; however, this was not the goal of our current studies. Additionally, this model is proposed for genetic susceptibility testing for common cancers (e.g. breast, colon, and ovarian) and may not apply in other contexts, such as genetic testing for rare cancers (e.g., pheochromocytomas), for which panels are commonly utilized and do not have the same wide variability in the disease spectrum, risks, or management options. Ultimately, there will need to be continued data collection and deliberation to guide best practices for delivery of cancer genetic testing as we transition from targeted to broader genomic applications. Equally important, the relative advantage of bundled versus discrete or limited genetic testing remains an overarching question that has been identified as a key research need in the era of genetic sequencing. ${ }^{16}$ Our model is not intended to address this larger question, but rather, given the current availability of clinical multiplex testing, to provide a potential delivery solution in this period of evaluation of the outcomes of multiplex testing including genes of variable clinical utility.

We are at a critical juncture in medicine, with the breadth and depth of available genetic information increasing exponentially and holding great promise for improving health outcomes. Multiplex genetic testing for cancer susceptibility raises the potential for greater complexity and uncertainty, and a tieredbinned approach to informed consent and genetic counseling has the potential to address these challenges. Given the current limitations and uncertainties, there is an urgent need to better understand the risks, benefits, and utilities of multiplex testing, and how to best deliver services and respect patient preferences as we debate how to implement genetic testing for the benefit of diverse patient populations and their families.

\section{SUPPLEMENTARY MATERIAL}

Supplementary material is linked to the online version of the paper at http://www.nature.com/gim

\section{ACKNOWLEDGMENTS}

This work was supported in part by grants from the National Cancer Institute (R01 CA160847), the American Society for Clinical Oncology Conquer Cancer Foundation, the Breast Cancer Research Foundation, and the Rooney Family Fund.

\section{DISCLOSURE}

Myriad Genetics paid for multiplex testing for participants in the METEOR study.

\section{REFERENCES}

1. Domchek SM, Friebel TM, Singer CF, et al. Association of risk-reducing surgery in BRCA1 or BRCA2 mutation carriers with cancer risk and mortality. JAMA 2010;304:967-975

2. Rainville IR, Rana HQ. Next-generation sequencing for inherited breast cancer risk: counseling through the complexity. Curr Oncol Rep 2014;16:371.

3. Goldgar DE, Healey S, Dowty JG, et al.; BCFR; KConFab. Rare variants in the ATM gene and risk of breast cancer. Breast Cancer Res 2011;13:R73.
4. Meijers-Heijboer $\mathrm{H}$, van den Ouweland A, Klijn J, et al.; CHEK2-Breast Cancer Consortium. Low-penetrance susceptibility to breast cancer due to CHEK2 $\left.{ }^{*}\right) 1100$ delC in noncarriers of BRCA1 or BRCA2 mutations. Nat Genet 2002;31:55-59.

5. Rahman N, Seal S, Thompson D, et al.; Breast Cancer Susceptibility Collaboration (UK). PALB2, which encodes a BRCA2-interacting protein, is a breast cancer susceptibility gene. Nat Genet 2007;39:165-167.

6. Lundy MG, Forman A, Valverde K, Kessler L. An investigation of genetic counselors' testing recommendations: pedigree analysis and the use of multiplex breast cancer panel testing. J Genet Couns 2014;23:618-632.

7. Stadler ZK, Schrader KA, Vijai J, Robson ME, Offit K. Cancer genomics and inherited risk. J Clin Oncol 2014;32:687-698.

8. Domchek SM, Bradbury A, Garber JE, Offit K, Robson ME. Multiplex genetic testing for cancer susceptibility: out on the high wire without a net? J Clin Oncol 2013;31:1267-1270.

9. Chong HK, Wang T, Lu HM, et al. The validation and clinical implementation of BRCAplus: a comprehensive high-risk breast cancer diagnostic assay. PLoS One 2014;9:e97408

10. Kurian AW, Hare EE, Mills MA, et al. Clinical evaluation of a multiplegene sequencing panel for hereditary cancer risk assessment. J Clin Oncol 2014:32:2001-2009.

11. Mauer CB, Pirzadeh-Miller SM, Robinson LD, Euhus DM. The integration of next-generation sequencing panels in the clinical cancer genetics practice: an institutional experience. Genet Med 2014;16:407-412.

12. Walsh T, Lee MK, Casadei $\mathrm{S}$, et al. Detection of inherited mutations for breast and ovarian cancer using genomic capture and massively parallel sequencing. Proc Natl Acad Sci USA 2010;107:12629-12633.

13. Hilbers FS, Vreeswijk MP, van Asperen CJ, Devilee P. The impact of next generation sequencing on the analysis of breast cancer susceptibility: a role for extremely rare genetic variation? Clin Genet 2013;84:407-414.

14. Burke W, Dimmock D. Clinical decisions. Screening an asymptomatic person for genetic risk. N Engl J Med 2014;370:2442-2445.

15. Bradbury AR, Patrick-Miller L, Domchek S. Multiplex genetic testing: reconsidering utility and informed consent in the era of next generation sequencing. Genet Med 2014; e-pub ahead of print 17 July 2014.

16. Presidential Commission for the Study of Bioethical Issues. Anticipate and Communicate: Ethical Management of Incidental and Secondary Findings in the Clinical, Research, and Direct-to-Consumer Contexts. Washington, DC, 2013.

17. Robson ME, Storm CD, Weitzel J, Wollins DS, Offit K; American Society of Clinical Oncology. American Society of Clinical Oncology policy statement update: genetic and genomic testing for cancer susceptibility. J Clin Oncol 2010;28:893-901.

18. Wolfe Schneider K, Anguiano A, Axell L, et al. Collaboration of colorado cancer genetic counselors to integrate next generation sequencing panels into clinical practice. J Genet Couns 2014;23:640-646.

19. Sharp RR. Downsizing genomic medicine: approaching the ethical complexity of whole-genome sequencing by starting small. Genet Med 2011;13:191-194.

20. Fecteau H, Vogel KJ, Hanson K, Morrill-Cornelius S. The evolution of cancer risk assessment in the era of next generation sequencing. J Genet Couns 2014;23:633-639.

21. Biesecker BB. Goals of genetic counseling. Clin Genet 2001;60:323-330.

22. Bernhardt BA, Biesecker BB, Mastromarino CL. Goals, benefits, and outcomes of genetic counseling: client and genetic counselor assessment. Am J Med Genet 2000;94:189-197.

23. Meiser B, Irle J, Lobb E, Barlow-Stewart K. Assessment of the content and process of genetic counseling: a critical review of empirical studies. J Genet Couns 2008; 17:434-451.

24. Robson ME, Storm CD, Weitzel J, Wollins DS, Offit K; American Society of Clinical Oncology. American Society of Clinical Oncology policy statement update: genetic and genomic testing for cancer susceptibility. J Clin Oncol 2010;28:893-901.

25. Trepanier AM, Allain DC. Models of service delivery for cancer genetic risk assessment and counseling. J Genet Couns 2014;23:239-253.

26. Elias S, Annas GJ. Generic consent for genetic screening. N Engl J Med 1994;330:1611-1613.

27. Riley BD, Culver JO, Skrzynia C, et al. Essential elements of genetic cancer risk assessment, counseling, and testing: updated recommendations of the National Society of Genetic Counselors. J Genet Couns 2012;21:151-161.

28. Berg JS, Khoury MJ, Evans JP. Deploying whole genome sequencing in clinical practice and public health: meeting the challenge one bin at a time. Genet Med 2011;13:499-504 


\section{ORIGINAL RESEARCH ARTICLE}

29. Ormond KE, Iris M, Banuvar S, Minogue J, Annas GJ, Elias S. What do patients prefer: informed consent models for genetic carrier testing. J Genet Couns 2007:16:539-550.

30. Bunnik EM, Janssens AC, Schermer MH. A tiered-layered-staged model for informed consent in personal genome testing. Eur J Hum Genet 2013;21:596601.

31. Patrick-Miller L. Development of a communication protocol for telephone disclosure of genetic test results for cancer predisposition. J Internet Res, in press.

32. Lammens CR, Aaronson NK, Wagner A, et al. Genetic testing in Li-Fraumen syndrome: uptake and psychosocial consequences. J Clin Oncol 2010;28:30083014.

33. Veach PM, Truesdell SE, LeRoy BS, Bartels DM. Client perceptions of the impact of genetic counseling: an exploratory study. J Genet Couns 1999;8:191-216.

34. Ellington L, Baty BJ, McDonald J, et al. Exploring genetic counseling communication patterns: the role of teaching and counseling approaches. J Genet Couns 2006;15:179-189.
BRADBURY et al | Tiered and binned genetic counseling model for multiplex testing

35. Ellington L, Maxwel A, Baty BJ, Roter D, Dudley WN, Kinney AY. Genetic counseling communication with an African American BRCA1 kindred. Soc SC Med 2007; 64:724-734

36. Garcia-Retamero R, Okan Y, Cokely ET. Using visual aids to improve communication of risks about health: a review. ScientificWorldJournal 2012;2012:562637.

37. Garcia-Retamero R, Galesic M. Who profits from visual aids: overcoming challenges in people's understanding of risks [corrected]. Soc Sci Med 2010;70:1019-1025

38. Hurle B, Citrin T, Jenkins JF, et al. What does it mean to be genomically literate? National Human Genome Research Institute Meeting Report. Genet Med 2013;15:658-663.

39. Ubel PA. Critical Decisions: How You and Your Doctor Can Make the Right Medical Choices Together. Harper Collins: New York, 2012.

40. Geller G, Botkin JR, Green MJ, et al. Genetic testing for susceptibility to adult-onset cancer. The process and content of informed consent. JAMA 1997:277:1467-1474. 